\title{
Illumina Sequencing
}

National Cancer Institute

\section{Source}

National Cancer Institute. Illumina Sequencing. NCI Thesaurus. Code C146817.

A proprietary next-generation DNA sequencing system from Solexa that uses reversible terminator nucleotides. The genomic DNA to be sequenced is fragmented and ligated to adapter molecules on both ends to construct an Illumina-specific adapter library. PCR amplification of the DNA fragments is then performed using the adapter sequence as primer. Sequencing is carried out by repeated cycles of adding reversible fluorescentlabelled nucleotide and incorporation of the nucleotides to the complementary strand. The fluorescence of the incorporated nucleotides is detected. 